Z. klin. Chem. u. klin. Biochem.

7. Jg., S. $514-517$, September 1969

\title{
Bestimmungen der Serumlipide mit Hilfe einer quantitativen eindimensionalen dünnschichtchromatographischen Methodik
}

\author{
Von J. Wildgrube, W. Erb und E. Böhle \\ Zentrum der Inneren Medizin der Universität Frankfurt|Main \\ (Direktoren: Prof. Dr. J. Frey, Prof. Dr. W. Siede, Prof. Dr. K. Schöffling, Prof. Dr. H. Martin)
}

(Eingegangen am 30. Mai 1969)

Es wird eine Methode zur eindimensionalen dünnschichtchromatographischen Trennung und quantitativen Auswertung der Serumlipidfraktionen beschrieben. Dieses Verfahren ermöglicht Aussagen über den Gehalt an Phospholipiden, Triglyceriden, Monoglyceriden, freien Fettsäuren, freiem Cholesterin und Cholesterinestern und genügt damit den Fragestellungen der Routinediagnostik. Die Ergebnisse von 20 Paralleluntersuchungen zu chemisch-analytischen Bestimmungen einzelner Lipidfraktionen werden verglichen und diskutiert.

\section{Determination of serum lipids by quantitative one dimensional thin layer cbromatography}

A method is described for the one dimensional thin layer chromatographic separation and quantitative evaluation of serum lipid fractions. The method gives values for phospholipides, triglycerides, monoglycerides, free fatty acids, free cholesterol and cholesterol esters, and it therefore satisfies the requirements for routine diagnosis. The results from 20 parallelstudies on the analytical chemical determination of individual lipid fractions are compared and discussed.

Dünnschichtchromatographische Lipidtrennungen haben auch für die klinische Diagnostik zunehmend an Bedeutung gewonnen. Zahlreiche Autoren konnten bisher über Gruppentrennungen verschiedener Serumlipide $(1-5)$ und weitergehendere Differenzierungen (6-13) berichten. Demgegenüber erforderten quantitative Auswertungen eine aufwendige Kombination mit kolorimetrischen $(9,14-16)$, spektrophotometrischen $(17,18)$ oder radiometrischen (19) Verfahren. Erst photodensitometrische Bestimmungen (20-22) und eine vereinfachende Methode durch direkte Extinktionsmessungen (23) konnten den apparativen und zeitlichen Aufwand vor allem bei der Bestimmung wenig polarer Lipide entscheidend reduzieren. In dieser Arbeit soll erstmals über eine analoge quantitative eindimensionale Trennung von Serumlipiden im Vergleich zu chemisch-analytischen Bestimmungen einzelner Lipidfraktionen berichtet werden.

\section{Methodik}

\section{Lipidextraktion}

Das Blut wird sofort nach der Entnahme auf $-1-2^{\circ}$ abgekühlt und nach Gerinnung im Kühlraum zentrifugiert. Man gibt $3 \mathrm{ml}$ Serum tropfenweise zu $50 \mathrm{ml}$ Äther-Äthanol 3:1 (v/v). Nach mindestens 8 Stdn. wird diese Lösung filtriert und die feinflockig ausgefallenen Proteine werden nochmals nachgewaschen. Der gesamte Serumlipidextrakt kann nun am Rotationsverdampfer eingeengt und zum Auftragen auf die DC-Platten verwendet werden. Die Bestimmung der Gesamtlipide erfolgt in einem zweiten Ansatz gravimetrisch gemäß den Angaben von ForcH und Mitarbeitern (24).

\section{Diinnscbichtchromatographie}

DC-Fertigplatten Kieselgel Merck $(5 \times 20 \mathrm{~cm})$ werden zunächst mit $n$-Hexan-Diäthyläther $1: 1(\mathrm{v} / \mathrm{v})$ vorgereinigt. Mit einer Sprühpistole kann der Lipidextrakt gleichmäßig aufgetragen werden; dabei ist die Kieselgelschicht durch eine Maske mit $2 \mathrm{~mm}$. breitem Spalt geschützt.

Als Fließmittel verwenden wir Chloroform-Methanol-Ameisensäure-Wasser $(65: 35: 2: 4 \mathrm{v} / \mathrm{v})$ - Fließmittel $\mathrm{I}$ - und n-Hexan-
Diạ̈thyläther-Eisessig $(80: 20: 1,5 \mathrm{v} / \mathrm{v})-$ Fließmittel II -. Die stark differierende Polarität der einzelnen Serumlipidfraktionen macht diese Fließmittelkombination notwendig. Dabei liegt der vorliegenden Stufentechnik folgendes Prinzip zugrunde: Fließmittel I verschiebt die Phospholipide vom Startpunkt und entwickelt die Fraktion der Monoglyceride. Die übrigen Lipidfraktionen wandern mit der Lösungsmittelfront und wērden erst durch Fließmittel II weiter differenziert in freies und verestertes Cholesterin, freie Fettsäuren und Triglyceride.

Jede Platte wird zunächst für $3 \mathrm{~cm}$ vom Auftrag mit ChloroformMethanol-Ameisensäure-Wasser entwickelt und bei Raumtemperatur getrocknet. Das zweite Fließmittel, n-Hexan-DiäthylätherEisessig, hat eine Laufstrecke von etwa $12 \mathrm{~cm}$. Damit ist eine gleichmäßige Verteilung der Fraktionen über die Platte gewährleistet.

Die Auftrennung der Serumlipide erfolgt mit steigenden $R_{F-}$ Werten in der Reihenfolge: Phospholipide, Monoglyceride, freies Cholesterin und Diglyceride, freie Fettsäuren, Triglyceride, Cholesterinester.

Wie Abbildung 1 erkennen läßt, werden die Phospholipide durch Fließmittel I vom Startpunkt verschoben und gespreizt. Vergleiche mit Testsubstanzen ${ }^{1}$ ) zeigten eine konstante Reihenfolge: Lysolecithin, Lecithin, Sphingomyelin und - bereits abgetrennt Kephalin. Während die Monoglyceride eine selbständige Fraktion bilden, läßt sich eine Kontamination der 1,2- und 1,3-Diglyceride mit der starken Cholesterinfraktion nicht vermeiden. Da Monound Diglyceride nur etwa 10\% der Triglyceride ausmachen (25), sind keine wesentlichen Ungenauigkeiten zu erwarten.

Die quantitative Auswertung erfolgt analog der direkten Extinktionsmessung von ERB und BöHLE (23). Hierzu werden die getrockneten Platten bei $180^{\circ}$ im Trockenschrank erhitzt, mit 20 proz. äthanolischer Phosphormolybdänsäure besprüht und für weitere 20 Min. im Trockenschrank belassen. Die einzelnen Fraktionen heben sich als blauschwarze Banden von der homogen gelbgrün gefärbten Platte ab (Abb. 2). Der oben beschriebene Vorgang hat sich als vorteilhaft erwiesen, um sämtliche Fraktionen vollständig darzustellen (26). Andererseits ist die vorgegebene Dauer des Erhitzens erforderlich, da die einzelnen Lipide unterschiedlich schnell verkohlen. Der Zeitbedarf nimmt zu in der Reihenfolge

1) Als Testsubstanzen verwandten wirir: 1-Monopalmitin, 1-Monoolein, 1,2-Dipalmitin, 1,3-Diolein, Tripalmitin, Lysolecithin und Sphingomyelin (Appl. Science Inc. Corp.), Cholesterin, CholesterylPalmitat, Triolein (Serva), DL- $\alpha$-Lecithin synth. und DL- $\alpha$-Kephalin synth. (Fluka). 
freies Cholesterin, Cholesterinester, Lecithin, Triglyceride, freie Fettsäuren und Monoglyceride. Abbildung 3 demonstriert diese Abweichung vom tatsächlichen Gehalt an einem Testgemisch mit gleichen Gewichtsanteilen von Lecithin, Palmitinsäure, Cholesterin und Cholesteryl-Palmitat.

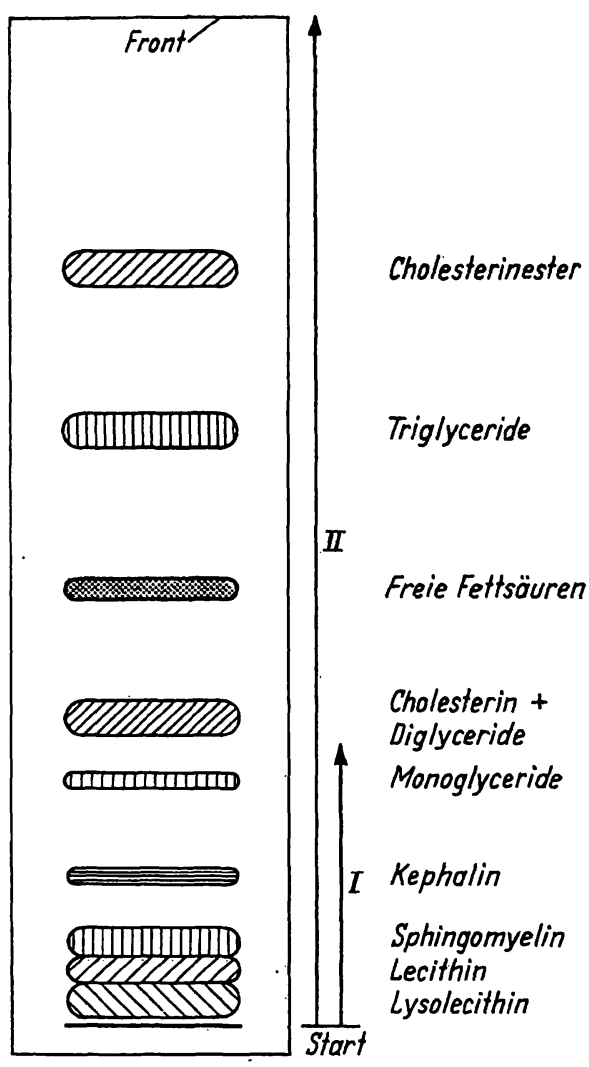

Abb. 1

Die Verteilung der einzelnen Lipidfraktionen nach optimaler Trennung durch Fließmittel I (Chioroform-Methanol-Ameisensäure-Wasse $65: 35: 2: 4 \mathrm{v} / \mathrm{v})$ und Fließmittel II (n-Hexan-Diäthyläther-Eisessig $80: 20: 1,5 \mathrm{v} / \mathrm{v})$
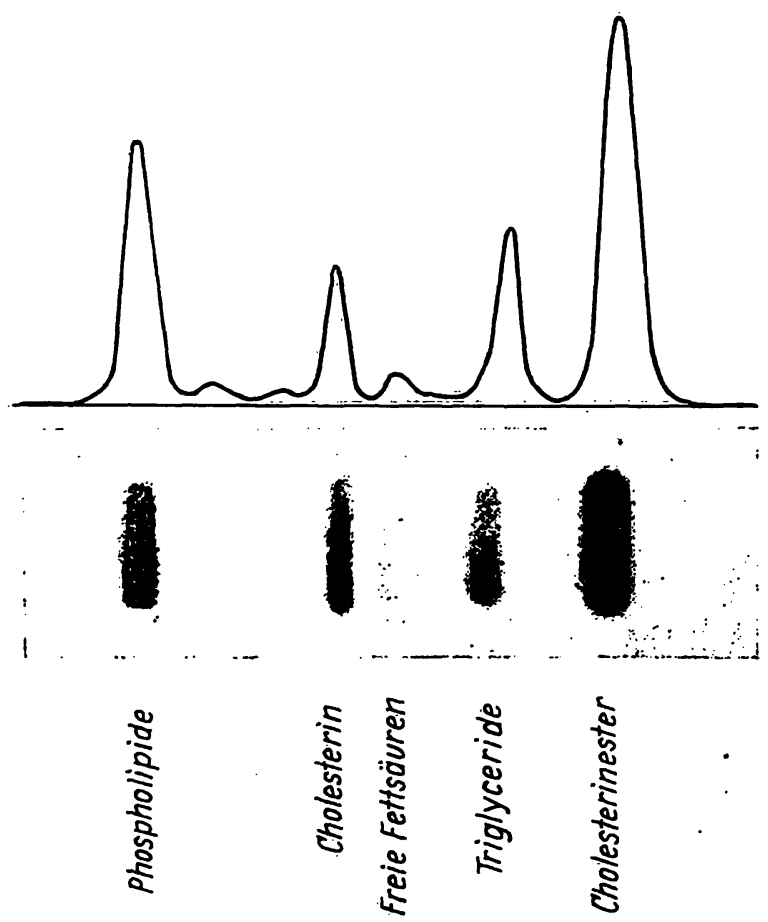

Abb. 2

Dünnschichtchromatogramm und Extinktionskurve der Serumlipide eines Gesunden

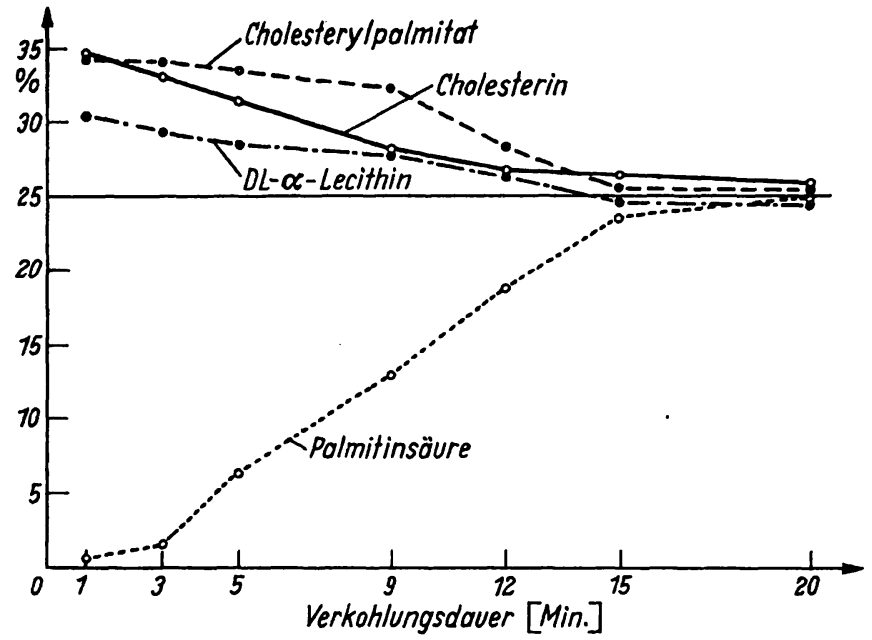

Abb. 3

Die Abhängigkeit der prozentualen Angaben von der Verkohlungsdauer bei einem Testgemisch mit gleichen Anteilen von Lecithin, Palmitinsäure, Cholesterin und Cholesteryl-Palmitat

Mit diesen Substanzen und Tripalmitin sowie Triolein wurde auch untersucht, ob mit zunehmenden Mengen der gleichen Substanz die Auswertungsergebnisse korrespondieren. Eine Linearität bleibt gewährleistet, solange die auswertbare Kurve hinreichend genau zu planimetrieren ist. Die homogen gelbgrüne Färbung der Platte hat keinen Einfluß auf das Verhältnis der einzelnen Fraktionen zueinander.

Die vom Extinktionsschreiber gezeichneten Kurven werden planimetriert und die prozentualen Anteile in Beziehung gesetzt zum gravimetrisch bestimmten Gesamtlipidgehalt.

\section{Ergebnisse und Diskussion}

Die Genauigkeit des vorliegenden Verfahrens liegt, gemessen an dem prozentualen Fehler, der auch Unterschiede beim Planimetrieren einschließt, mindestens im Bereich unserer übrigen chemisch-analytischen Methoden. Der aus 20 Doppelbestimmungen ermittelte methodische Fehler $\left(\mathrm{m}=\sqrt{\frac{\Sigma^{\prime}\left(\mathrm{x}_{1}-\mathrm{x}_{2}\right)^{2}}{2 \mathrm{n}}}\right)$ beträgt für die Phospholipide bei einem Mittelwert von (28,2 [rel.-\%]) $\pm 1,9 \%$, für freie Fettsäuren $(1,8) \pm 0,3 \%$, Triglyceride $(19,3) \pm 1,9 \%$, für freies Cholesterin $(10,3) \pm 0,9 \%$ und für Cholesterinester $(40,9) \pm 2,1 \%$.

Durch das Kalt-Extraktionsverfahren mit Äther-Äthanol lassen sich die Serumlipide nahezu quantitativ isolieren. Bei einer nochmaligen Extraktion des Eiweißrückstandes mit Chloroform-Methanol nach Folch und Mitarbeitern (24) wurden stets weniger als 5\% der Gesamtlipidmenge gefunden. Darüber hinaus vermindert die Kaltextraktion eine Alteration einzelner Lipide wie wir sie unter Chloroform-Methanol sahen (Freisetzung von Fettsäuren und Umesterungen zu Fettsäurenmethylestern, wobeinicht nur Triglyceride, sondern auch Cholesterinester und Phospholipide betroffen sind). Enzymatisch bedingte Lipolysen können durch das Abkühlen des Blutes sofort nach der Entnahme stark reduziert oder durch lipasehemmende Stoffe (wie $p$ Nitrophenyldiaethylorthophosphat [Mintacol], in einer Konzentration von $200 \mathrm{mg} / 100 \mathrm{ml}(27,28)$ ) unmittelbar gestoppt werden.

Demgegenüber ist die Umrechnung auf absolute Werte von einer exakten Gesamtlipidbestimmung abhängig. 
Hier führen nur strengste Kautelen zu befriedigenden Doppelbestimmungen.

Die Ergebnisse von Paralleluntersuchungen zu 20 im Routinelabor bestimmten Fettstaten sind in Tabelle 1 und 2 zusammengefaßt. Dabei wurden die Phospholipide als anorganischer Phosphor in einer Modifikation von BARTLETT (29), die Neutralfette nach EGGSTEIN und KREUTZ (30), nicht veresterte Fettsäuren nach MosinGER (31) und das Gesamtcholesterin entsprechend den Angaben der Fa. Hyland nach SeArCy und Bergquist (32) bestimmt. Die Gegenüberstellung dieser und der dünnschichtchromatographisch ermittelten Werte zeigt, $\mathrm{da} B$ die Differenzen im Bereich des methodischen Fehlers liegen. Die Regression ist linear; der Koeffizient $\left(\mathrm{b}=\frac{\Sigma^{\prime} \mathrm{x} \cdot \mathrm{y}-\overline{\mathrm{y}} \Sigma^{\prime} \mathrm{x}}{\Sigma^{\prime} \mathrm{x}^{2}-\overline{\bar{x}} \Sigma^{\prime} \mathrm{x}}\right)$ beträgt für die Bestimmungen der Phospholipide 0,97, der Neutralfette (Dünnschicht: Mono- und Triglyceride) 0,87 und des Gesamtcholesterins 0,94. Entscheidende Unterschiede ergeben sich in den Angaben der freien Fettsäuren; konstante Korrelationen sind hier nicht zu berechnen. Andererseits läßt. der nur wenig variierende prozentuale Anteil der freien Fettsäuren (Tab. 2) einen anderen Normbereich bei der dünnschichtchromatographischen Trennung vermuten.
Der chemisch-analytischen Gesamt-Cholesterinangabe stehe zwei Werte gegenüber, die den Gehalt an freiem Cholesterin und Chölesterinestern (Cholesterin + Fettsäure) bestimmen. Unter der Voraussetzung, daß mit dem Cholesterin vorwiegend Fettsäuren mit $18 \mathrm{C}$ Atomen verestert sind (33-35), kann der Gesamtcholesteringehalt errechnet werden; es gilt:

$$
\begin{gathered}
\text { Gesamt-Cholesterin }=\text { freies Cholesterin } \\
+(\text { Cholesterinester } \cdot 0,59) .
\end{gathered}
$$

Die prozentuale Aufschlüsselung der Lipidfraktionen zeigt bei Gesunden und Patienten mit erhöhten Serumlipidwerten erhebliche Schwankungsbreiten, die im einzelnen noch nicht zu deuten sind. Dazu zählen auch die unterschiedlich hohen Anteile des freien Cholesterins. Auf dieses Phänomen haben bereits Leonard und Mitarbeiter (36) nachdrücklich hingewiesen. Entsprechende Unterschiede konnten wir auch bei Säuglingen unter verschiedenen Nahrungsregimen beobachten (37). Die vorliegende Methode ist gut für die Routinediagnostik geeignet, da sie mit relativ geringem Aufwand eine differenzierte Analyse der Serumlipide ermöglicht. Statt der angegebenen können auch mit Kieselgel G nach STAkL selbst beschichtete Platten nach

Táb. 1

Gegenüberstellung der dünnschichtchromatographisch (dc) und chemisch-analytisch (ca) ermittelten Serumlipid-Fraktionen (Angaben in mg/

\begin{tabular}{|c|c|c|c|c|c|c|c|c|c|c|}
\hline Nr. & \multicolumn{2}{|c|}{$\begin{array}{l}\text { Phospholipide } \\
\text { dc ca }\end{array}$} & \multicolumn{2}{|c|}{$\begin{array}{l}\text { Neutralfette } \\
\text { dc ca }\end{array}$} & $\begin{array}{r}\text { frèie } \\
\text { dc }\end{array}$ & $\begin{array}{c}\text { Fettsäuren } \\
\text { ca }\end{array}$ & \multicolumn{2}{|c|}{$\begin{array}{c}\text { Gesamtcholesterin } \\
\mathrm{dc}\end{array}$} & $\begin{array}{c}\text { freies Cholesterin } \\
\text { dc }\end{array}$ & $\begin{array}{c}\text { Cholesterinester } \\
\text { dc }\end{array}$ \\
\hline $\begin{array}{r}1 \\
2 \\
3 \\
4 \\
5 \\
6 \\
7 \\
8 \\
9 \\
10 \\
11 \\
12 \\
13 \\
14 \\
15 \\
16 \\
17 \\
18 \\
19 \\
20\end{array}$ & $\begin{array}{l}287 \\
260 \\
292 \\
338 \\
237 \\
316 \\
309 \\
255 \\
192 \\
276 \\
179 \\
217 \\
189 \\
118 \\
220 \\
191 \\
450 \\
354 \\
607 \\
321\end{array}$ & $\begin{array}{l}301 \\
264 \\
284 \\
360 \\
234 \\
336 \\
306 \\
240 \\
238 \\
304 \\
202 \\
237 \\
203 \\
125 \\
226 \\
206 \\
442 \\
385 \\
635 \\
325\end{array}$ & $\begin{array}{r}84 \\
215 \\
189 \\
122 \\
201 \\
177 \\
163 \\
146 \\
143 \\
184 \\
259 \\
133 \\
170 \\
155 \\
134 \\
222 \\
290 \\
160 \\
383 \\
178\end{array}$ & $\begin{array}{l}101 \\
238 \\
174 \\
145 \\
202 \\
198 \\
129 \\
155 \\
119 \\
199 \\
241 \\
124 \\
193 \\
150 \\
122 \\
245 \\
279 \\
145 \\
362 \\
158\end{array}$ & $\begin{array}{r}14 \\
6 \\
23 \\
6 \\
10 \\
16 \\
18 \\
10 \\
12 \\
18 \\
22 \\
55 \\
31 \\
15 \\
10 \\
15 \\
33 \\
24 \\
37 \\
14\end{array}$ & $\begin{array}{r}584 \\
388 \\
1060 \\
310 \\
480 \\
590 \\
614 \\
502 \\
600 \\
650 \\
797 \\
258 \\
1160 \\
785 \\
436 \\
610 \\
1270 \\
961 \\
1090 \\
675\end{array}$ & $\begin{array}{l}270 \\
251 \\
293 \\
291 \\
342 \\
253 \\
213 \\
210 \\
205 \\
294 \\
254 \\
179 \\
200 \\
205 \\
169 \\
200 \\
385 \\
437 \\
432 \\
288\end{array}$ & $\begin{array}{l}260 \\
241 \\
265 \\
306 \\
318 \\
287 \\
248 \\
215 \\
203 \\
256 \\
210 \\
180 \\
185 \\
195 \\
180 \\
185 \\
330 \\
420 \\
398 \\
255\end{array}$ & $\begin{array}{r}83 \\
50 \\
97 \\
87 \\
109 \\
49 \\
120 \\
46 \\
63 \\
124 \\
66 \\
58 \\
51 \\
66 \\
34 \\
67 \\
83 \\
146 \\
158 \\
119\end{array}$ & $\begin{array}{r}317 \\
350 \\
332 \\
346 \\
395 \\
347 \\
362 \\
278 \\
240 \\
.289 \\
320 \\
204 \\
254 \\
236 \\
230 \\
225 \\
511 \\
493 \\
465 \\
488\end{array}$ \\
\hline
\end{tabular}
$100 \mathrm{ml}$ außer den chemisch-analytisch bestimmten Werten der freien Fettsäuren $(\mu \mathrm{V}$ al $/ l)$

Tab. 2

\begin{tabular}{|c|c|c|c|c|c|c|c|}
\hline Nr. & $\begin{array}{c}\text { Gesamtlipide } \\
\mathrm{mg} / 100 \mathrm{ml}\end{array}$ & $\begin{array}{c}\text { Phospholipide } \\
\%\end{array}$ & $\underset{\%}{\text { Neutralfette }}$ & $\begin{array}{c}\text { freie } \\
\text { Fettsäuren } \\
\%\end{array}$ & $\begin{array}{c}\text { freies } \\
\text { Cholesterin } \\
\%\end{array}$ & $\begin{array}{c}\text { Cholesterin- } \\
\text { ester } \\
\%\end{array}$ & $\begin{array}{c}\text { Gesamt- } \\
\text { cholesterin } \\
\%\end{array}$ \\
\hline $\begin{array}{r}1 \\
2 \\
3 \\
4 \\
5 \\
6 \\
7 \\
8 \\
9 \\
10 \\
11 \\
12 \\
13 \\
14 \\
15 \\
16 \\
17 \\
18 \\
19 \\
20\end{array}$ & $\begin{array}{l}785 \\
881 \\
933 \\
899 \\
952 \\
905 \\
972 \\
735 \\
650 \\
891 \\
846 \\
617 \\
695 \\
590 \\
628 \\
720 \\
1367 \\
1177 \\
1650 \\
1120\end{array}$ & $\begin{array}{l}36,5 \\
29,5 \\
31,3 \\
37,6 \\
24,9 \\
34,9 \\
31,8 \\
34,7 \\
29,5 \\
31,0 \\
21,1 \\
35,3 \\
27,2 \\
20,0 \\
35,0 \\
26,5 \\
32,9 \\
30,1 \\
36,8 \\
28,7\end{array}$ & $\begin{array}{l}10,7 \\
24,4 \\
20,3 \\
13,6 \\
21,1 \\
19,6 \\
16,8 \\
19,9 \\
22,0 \\
20,6 \\
30,6 \\
21,6 \\
24,5 \\
26,2 \\
21,3 \\
30,8 \\
21,2 \\
13,6 \\
23,2 \\
15,9\end{array}$ & $\begin{array}{l}1,8 \\
1,3 \\
2,4 \\
0,6 \\
1,0 \\
1,8 \\
1,8 \\
1,4 \\
1,9 \\
2,1 \\
2,7 \\
1,4 \\
2,1 \\
2,6 \\
1,7 \\
2,1 \\
2,4 \\
2,0 \\
2,1 \\
1,2\end{array}$ & $\begin{array}{r}10,6 \\
5,1 \\
10,4 \\
9,7 \\
11,5 \\
5,4 \\
12,4 \\
6,2 \\
9,7 \\
13,9 \\
7,8 \\
8,7 \\
7,7 \\
11,2 \\
5,4 \\
9,3 \\
6,1 \\
12,4 \\
9,6 \\
10,6\end{array}$ & $\begin{array}{l}40,4 \\
39,7 \\
35,6 \\
38,5 \\
41,5 \\
38,3 \\
37,2 \\
37,8 \\
36,9 \\
32,4 \\
37,8 \\
33,0 \\
38,5 \\
40,0 \\
36,6 \\
31,3 \\
37,4 \\
41,9 \\
28,3 \\
43,6\end{array}$ & $\begin{array}{l}34,4 \\
28,5 \\
31,4 \\
32,4 \\
35,9 \\
27,9 \\
21,9 \\
28,6 \\
31,5 \\
33,0 \\
30,0 \\
29,0 \\
28,8 \\
34,7 \\
26,9 \\
27,8 \\
28,2 \\
37,1 \\
26,2 \\
25,7\end{array}$ \\
\hline
\end{tabular}

Gesamtlipide und die dünnschichtchromatographisch ermittelten prozentualen Anteile der Serumlipidfraktionen 
Verkohlen mit Schwefelsäure (Methodisches bei ERB und Börre (23)) ausgewertet werden. Freilich bleibt zu berücksichtigen, daß die Stufentechnik bei diesen Platten eine Vorreinigung wegen der empfindlichen Kieselgelschicht unmöglicht macht. Damit muß eine Kontamination der Cholesterinester-Fraktion mit der Schmutzfront in Kauf genommen werden. Darüber hinaus erfordert dieses Verfahren neben sehr sorgfältig beschichteten Platten einen höheren Zeitaufwand beim Verkohlen.

Die Untersuchungen wurden mit Unterstützung der Deutschen Forschungsgemeinschaft (Bo 150/10) durchgeführt. Frl. M. WILHELM danken wir für wertvolle Mitarbeit.

\section{Literatur}

1. Wercker, H., Klin. Wschr. 37, 763 (1959). - 2. Vogel, W. C., W. M. DoIzakI und L. ZIEve, J. Lipid Res. 3, 138 (1962). 3. Sachs, B. A. und L. Wolfman, Proc. Soc. Exp. Biol. Med. N. Y. 115, 1138 (1964). - 4. Schlierf, G. und P. Wood, J. Lipid Res. 6, 317 (1965). - 5. PIE, A. und A. GINER, Nature (London) 212, 402 (1966). - 6. ZölLNER, N., G. Wolfram und G. AmIN, Klin. Wschr. 40, 273 (1962). - 7. HabermanN, E., G. Bandtrow und B. Krusche, Klin. Wschr. 39, 816 (1961). 8. WAGENER, H., D. LANG und B. FrosCh, Zschr. ges. Exp. Med. 138, 425 (1964). - 9. Amenta, J. S., J. Lipid Res. 5, 270 (1964). 10. WAGENER, H., Nature (London) 205, 386 (1965). - 11. KAUfmanN, H. P., S. S. Radwan und A. K. S. Almad, Fette, Seifen, Anstrichmittel 68, 261 (1966). - 12. FreEMAN, C. P. und D. West, J. Lipid Res. 7, 324 (1966). - 13. ZAhLER, P., diese Z. 5, 191 (1967). - 14. Vioque, E. und L. J. Morris, J. Amer. Oil Chemists' Soc. 38, 482 (1961). - 15. Robinson, N. und B. M. PhiLlips, Clin. Chim. Acta Amsterdam 8, 385 (1963). - 16. Mangold, H. K., J. Amer. Oil Chemists' Soc. 41, 762 (1964). - 17. KRELI, K. und S. A. Hashim, J. Lipid Res. 4, 407 (1963). - 18. GäNshirt, H., F. W. Koss und W. K. Morianz, Arzneimittelforsch. (Aulendorf) 10, 943 (1960). - 19. SNYDER, F. und N. STEPhENS, Biochem. biophysica Acta Amsterdam 39, 244 (1959). - 20. PrIVetT, O. S., M. L. Blank und W. O. LundBerg, J. Amer. Oil Chemists' Soc. 38, 312 (1961). - 21. PAYNe, S. N., J. Chromato- graphy 15, 173 (1964). - 22. Privetr, O. S., M. L. Blank, D. W. Codding und E. C. Nickell, J. Amer. Oil Chemists' Soc. 42, 381 (1965). - 23. ERB, W. und E. BöHLE, diese Z. 6, 379 (1968). 24. Folch, J., M. Lees und G. H. Sloane Stanley, J. Lipid Res. 1, 391 (1959). - 25. Vogelberg, K. H. und F. A. Gries, Fette, Seifen, Anstrichmittel 70, 851 (1968). - 26. Hofman, A. F., Thin-layer adsorption chromatography of lipids in 'Biochemical problems of lipids' ed. by A. C. Frazer, Elsevier Publishing Comp. Amsterdam (1963). - 27. Elmendorf, H. von und D. VAlleE, Blut 10, 61 (1964). - 28. EhrLy, A. M., Biorheology 5, 209 (1968). - 29. Bartlett, G. R., J. biol. Chemistry 234, 466 (1959). - 30. Eggstein, M. und F. H. Kreutz, Enzymatische Glycerinbestimmung und Neutralfettberechnung, in , Untersuchungen und Bestimmung der Lipoide im Blut hrsg. von N. Zöllner und D. Eberhagen, Springer-Berlin (1965). - 31. Mostnger, F., J. Lipid Res. 6, 157 (1965). - 32. Searcy, R. L. und L. M. Bergquist, Clin. Chim. Acta Amsterdam 5, 192 (1960). 33. Schrade, W., E. Böhle, R. Biegler, R. Teicke und B. UllRICH, Klin. Wschr. 43, 739 (1960). - 34. Böhle, E., E. Harmuth und M. Scriba, Gastroenterologia, Suppl. ad Vol. 104, 254 (1965). 35. Goodman, D. S., Physiol. Rev. 45, 747 (1965). - 36. Leonard, P. J., A. G. Shaper und K. W. Jones, Amer. J. Clin. Nutr. 17, 377 (1965). - 37. Wildgrube, J., Fette, Seifen, Anstrichmittel 70,852 (1968).
Prof. Dr. E. Böhle 493 Detmold Lagesche Str. 47 